\title{
New $\delta$ Scuti variable in the Pleiades: HD 23628
}

\author{
Z. P. Li ${ }^{1}$, E. Michel ${ }^{2}$, L. Fox Machado ${ }^{3}$, M. Álvarez ${ }^{4}$, M. M. Hernández ${ }^{3}$, M. Chevreton ${ }^{2}$, A. Y. Zhou ${ }^{1}$, C. Barban ${ }^{2,7}$, \\ N. Dolez ${ }^{5}$, J. A. Belmonte ${ }^{3}$, A. Fernandez ${ }^{2}$, J. Fremy ${ }^{2}$, S. Pau ${ }^{2}$, B. Servan ${ }^{6}$, F. Pérez Hernández ${ }^{3,8}$, S. Y. Jiang ${ }^{1}$, \\ Z. L. Liu ${ }^{1}$, O. Ringot ${ }^{2}$, J. C. Suárez ${ }^{2}$, and Y. Xu ${ }^{1}$
}

1 National Astronomical Observatories, Chinese Academy of Sciences, Beijing 100012, China

2 Observatoire de Paris, LESIA, FRE 2461, 92195 Meudon, France

${ }^{3}$ Instituto de Astrofísica de Canarias, 38200 La Laguna, Tenerife, Spain

${ }^{4}$ Instituto de Astronomía-UNAM, Ap.P. 877, Ensenada, BC, Mexico

5 Observatoire Midi-Pyrénées, UMR CNRS, 31400 Toulouse, France

${ }^{6}$ Observatoire de Paris, GEPI, FRE2459, 92195 Meudon, France

7 National Solar Observatory, Tucson, AZ 85719, USA

8 Departamento de Astrofísica, Universidad de La Laguna, Tenerife, Spain

Received 15 June 2001 / Accepted 28 August 2002

\begin{abstract}
We report here the discovery of very low amplitude oscillations in the Pleiades star HD 23628. The 14-nights high quality light curve designates HD 23628 as a new member of the $\delta$ Scuti stars class. Amplitude spectra indicate a multi-periodic pulsation behaviour, and four pulsation frequencies have been detected so far. The frequency distribution suggests the presence of nonradial modes. Pulsation constant values of the four modes are distributed in the range corresponding to fundamental $f$ to $p_{4}$ radial modes.
\end{abstract}

Key words. stars: oscillations - stars: individual: HD 23628 - stars: variables: $\delta$ Sct

\section{Introduction}

Seismology of $\delta$ Scuti stars is expected to bring fruitful constraints on the current understanding and modelling of intermediate-mass stars at the stage of the Main-sequence evolution and thick-shell hydrogen burning. The strong interest in these objects has motivated great observational efforts. A recent review by Rodríguez et al. (2000) revealed more than 700 known $\delta$ Scuti stars. Among these objects, stars in open clusters are of special interest, in terms of theoretical interpretation, for the additional constraints they bring on age, chemical composition, distance, etc. The case of the Praesepe cluster, with $14 \delta$ Scuti members, is very illustrative of this observational effort (Breger 1973; Álvarez et al. 1998; Hernández et al. 1998).

In this respect, the Pleiades cluster is also an attractive candidate and, because of its young age compared to Praesepe, it is expected to provide a valuable complementary point of view on the problem. In Pleiades, $5 \delta$ Scuti were known so far and all of them have been programmed as target stars for the STEPHI network in recent years (see e.g. Fox-Machado et al. 2002). The theoretical interpretation of these results in terms of stellar structure and evolution is already under way (Suárez et al. 2001).

The discovery of a new $\delta$ Scuti star in the Pleiades is reported here. In Sect. 2, we briefly introduce HD 23628.

Send offprint requests to: Z.-P. Li, e-mail: lizhi@bao.ac.cn
In Sect. 3, the observing conditions and the data obtained for this object are described. The data reduction and data analysis are discussed in Sect. 4. Finally, Sect. 5 is devoted to conclusions.

\section{The object HD 23628}

HD 23628 is a member of Pleiades with $m_{v}=7.68$ and spectral class A4V. Crawford \& Perry (1976) determined its $u v b y-\mathrm{H} \beta$ indices as: $b-y=0.125, m_{1}=0.183, c_{1}=0.910$ and $\beta=2.856$. According to Hipparcos individual parallax measurements of cluster members, Van Leeuwen (1999) proposed a $5.37 \pm 0.07$ estimate of the distance modulus for the Pleiades. Following this value and considering the precision required in the framework of this study, we adopt $M_{v}=2.3 \mathrm{mag}$ for HD 23628. This value is in reasonable agreement with other values proposed for the distance modulus (5.59 \pm 0.12 Gatewood et al. 2000) as well as with estimates obtained via the $M_{v}$ calibration proposed by Crawford (1979) $\left(M_{v}=2.276 \pm 0.2\right)$. For effective temperature and gravity, we adopt the values $T_{\text {eff }}=8120 \mathrm{~K}$ and $\log g=4.07$ estimated from $u v b y-\mathrm{H} \beta$ photometry, following Moon \& Dworetsky (1985).

HD 23628 has already been searched for variability in the framework of a survey in the Pleiades (Breger 1972). Despite a mean dispersion of the order of $0.002 \mathrm{mag}$, no obvious variation was found in these observations. In the light of our new data, this has to be attributed to the shortness (2.2 hours) of the time series collected by Breger (1972). 
Table 1. The log of the observations of HD 23628.

\begin{tabular}{cccc}
\hline \hline Day & Date 1998 & Start Time (HJD) & End Time (HJD) \\
\hline 1 & Nov. 12-13 & 130.42 & 130.63 \\
2 & Nov. 13-14 & 131.37 & 131.75 \\
3 & Nov. 14-15 & 132.39 & 132.74 \\
4 & Nov. 15-16 & 133.39 & 133.74 \\
5 & Nov. 16-17 & 134.38 & 134.72 \\
6 & Nov. 17-18 & 135.43 & 135.74 \\
7 & Nov. 18-19 & 136.38 & 136.74 \\
8 & Nov. 19-20 & 137.38 & 137.74 \\
9 & Nov. 20-21 & 138.50 & 138.73 \\
10 & Nov. 21-22 & 139.38 & 139.73 \\
11 & Nov. 22-23 & 140.37 & 140.73 \\
12 & Nov. 23-24 & 141.35 & 141.72 \\
13 & Nov. 24-25 & 142.35 & 142.72 \\
14 & Nov. 25-26 & 143.36 & 143.68 \\
\hline
\end{tabular}

\section{The observations}

The star HD 23628 was chosen as a second comparison star at the Observatorio del Teide (Tenerife, Spain) during the STEPHI observations in November 1998. This star was hitherto supposed constant and its spectral type and position near our main target HD 23567 made it a suitable comparison. As a result, more than 110 hours of rapid photometry data of HD 23628 have been collected from this site, over approximately 2 weeks, using $1.5 \mathrm{~m}$ Carlos Sánchez Telescope. The log-book of these observations is given in Table 1. The time sampling is $1 \mathrm{~s}$ and the filter used is Strömgren $v$ (interferometric filters with $\lambda \sim 4200 \AA, \Delta \lambda \sim 190 \AA$ ).

From the same observational runs, similar data have been collected for the background sky contribution and for the star HD $23627\left(m_{v}=8.5, \mathrm{~B} 8 \mathrm{~V}\right.$, see respectively Cvetkovic 1992 and Roeser \& Bastian 1988), which has been used here as comparison star in the data reduction presented in Sect. 4. All these data have been collected simultaneously, using the Chevreton multi-channel photometer (see e.g. Michel et al. 1995).

These time series are remarkable in the first place for the quantity of data obtained from one site over the corresponding period. With approximately 112 hours of data over 14 days, we are very near the one-site optimal coverage. In addition to this and as mentioned in Sect. 4.2, the average quality of these data is high according to present standards, allowing detection of oscillation peaks below $1 \mathrm{mmag}$.

\section{Data reduction and data analysis}

\subsection{Data reduction}

The data have been reduced following the classical data reduction scheme for multi-channel rapid photometry. The time series corresponding to sky background contribution was first subtracted from the time series associated respectively with HD 23628 and HD 23627. This was done taking into account sensitivity ratios between the channel dedicated to the sky background and each of the two other channels. These sensitivity ratios are determined from sky-flat sequences, one at the beginning of the night and one at the end, when all the different

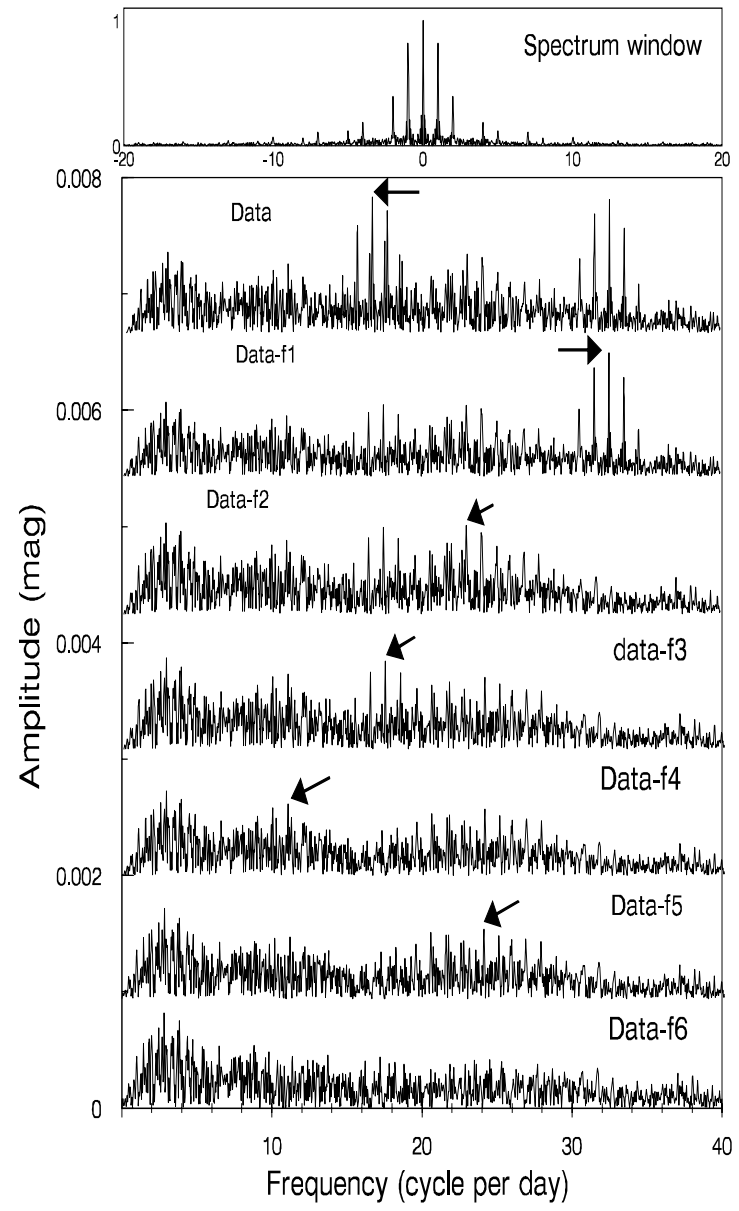

Fig. 1. The amplitude spectra of HD 23628 which include all the data. The spectra are shown before and after applying multiple frequency solution.

channels are observing simultaneously twilight sky. The sensitivity ratio at a given time is obtained by a linear interpolation in time between these two values.

The two resulting time series associated with each star were then divided one by the other (HD 23628/HD 23627) in order to obtain a differential photometry time series free of extinction effects.

At this point, some low frequency noise remains in the data. This can be attributed mainly to small relative drifts in the sensitivity of photomultipliers of different channels, to residual colour effects in the extinction correction (although stars are taken very close spatially on the sky and relatively close in spectral type). This kind of noise is filtered by dividing nightly differential time series by a low degree $(\leq 2)$ polynomial fit.

The data are then merged together. The time is converted to Heliocentric Julian time and resampled with a 1 minute time sampling.

\subsection{Data analysis}

The Fourier spectrum of the differential time series resulting from the previous data reduction is shown in Fig. 1. Oscillation peaks can be seen in the 15 to 35 cycles per day range (100-400 $\mu \mathrm{Hz}$ range). An analysis of these data has been done 

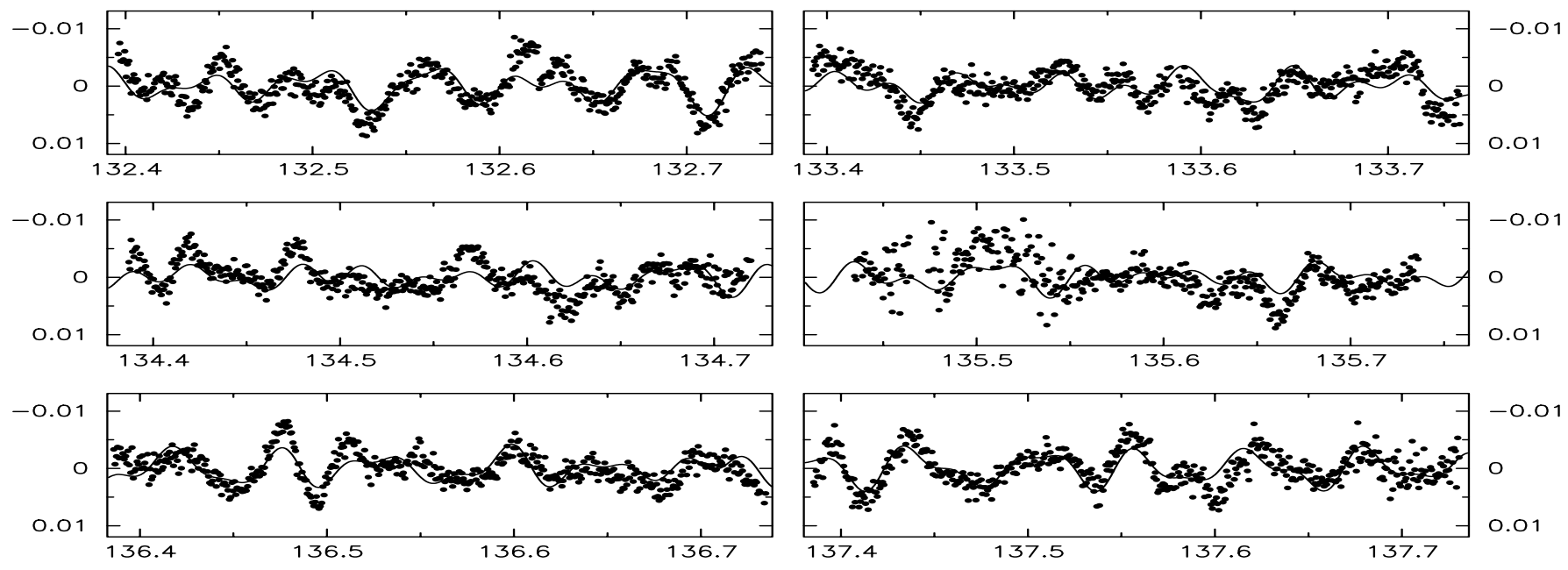

Fig. 2. The fit of the six-frequency solution to part of the data. Abscissa is the time in HJD 2451000+ days, ordinate in magnitudes.

Table 2. Results of the data analysis.

\begin{tabular}{|c|c|c|c|c|}
\hline \multicolumn{3}{|c|}{ Frequency } & \multirow{2}{*}{$\begin{array}{c}\text { Amplitude } \\
v \text { Filter } \\
\text { mmag }\end{array}$} & \multirow{2}{*}{$\begin{array}{c}\text { Significance } \\
\text { Amplitude } S / N\end{array}$} \\
\hline Name & $\mathrm{cd}^{-1}$ & $\mu \mathrm{Hz} / \mathrm{ID}$ & & \\
\hline$f_{1}$ & 16.59 & 192.0 & 1.29 & 9.2 \\
\hline$f_{2}$ & 32.54 & 376.7 & 1.37 & 11.4 \\
\hline$f_{3}$ & 22.96 & 265.7 & 0.87 & 4.0 \\
\hline$f_{4}$ & 17.42 & 201.6 & 0.81 & 4.8 \\
\hline$f_{5}$ & 10.93 & 126.5 & 0.69 & 3.3 \\
\hline$f_{6}$ & 24.04 & 277.2 & 0.68 & 3.5 \\
\hline
\end{tabular}

following the method described by Li et al. (1991), using the package Period98 (Sperl 1998).

Figure 1 shows the amplitude spectra of the data before and after subtraction of the six best oscillation frequency candidates. The comparison of this six-frequency solution to the data is illustrated in Fig. 2. The spectral window associated with this time series exhibits the classical $1 \mathrm{c} / \mathrm{d}$ aliases with reasonable amplitudes for one-site observations, the first alias being approximately $80 \%$ of the central peak in amplitude (see top of Fig. 1). The results of this analysis are given in Table 2.

Peaks 1 to 4 are detected with signal-to-noise values higher than or equal to 4 . Their detection can thus be attributed a 99\% confidence level according to the Fisher's test (see, e.g. Nowroozi 1967 and Michel et al. 1992). The other two peaks show slightly lower amplitudes. Their detection has to be considered with more caution. However, discarding these two peaks degrades the fit to the data as can be seen in Fig. 3. The noise level mentioned in the last column of Table 2 has been estimated as a function of frequency after subtraction of the best six frequencies. The values are not significantly changed if only the four ascertained frequencies are subtracted. The mean noise level remains lower or equal to $2 \times 10^{-4}$ in amplitude over the range of frequency of interest here. This corresponds to a standard deviation of $\sigma=7 \times 10^{-2}$ per 1 second integration, in the time series. This mean value obtained over 112 hours is satisfactory when a rough estimate, taking into account photon noise and standard scintillation only, leads to an expected $\sigma=1 \times 10^{-2}$.

Considering the range of frequency represented in Table 2 and HD 23628 being located in the low instability strip, we conclude that this object is a new low amplitude $\delta$ Scuti star.

HD 23627 is given as a B8 field star in the PPM catalogue (Roeser et al. 1988). Short period pulsations as those considered here have never been announced for such an object. This suggests that all the previous oscillation peaks are due to HD 23628, even if it cannot be taken as a definitive proof. Ideally, the simultaneous observation of a third star is the way to settle this point. Unfortunately, in the present case, HD 23567 (a known $\delta$ Scuti star which has been observed simultaneously) can hardly play this role, because its own oscillation peaks, located in the same domain with significantly larger amplitudes than the ones mentioned in Table 2, dominate the spectra.

Indicative values of the pulsation constants $Q$ can be estimated following the relation (Breger 1990):

$\log Q=-6.454+\log P+0.5 \log g+0.1 M_{\mathrm{bol}}+\log T_{\mathrm{eff}}$.

Using the values discussed in Sect. 2 for $M_{\mathrm{bol}}, \log T_{\mathrm{eff}}$ and $\log g$, we find that the $Q$ values for $f_{1}$ to $f_{4}$ are $0.032 \pm$ $0.0058,0.016 \pm 0.0029,0.023 \pm 0.0041$ and $0.030 \mathrm{~d} \pm 0.0054$ respectively, where an uncertainty of $18 \%$ is adopted (Breger 1990). According to these values, corresponding pulsation modes would be distributed in the range from $f$ to $p_{4}$ mode. We notice that these modes have to feature at least one nonradial mode, since frequencies $f_{1}$ and $f_{4}$ are too close to be both radial modes, even in the presence of fast rotation $(V \sin i \sim$ $200 \mathrm{~km} \mathrm{~s}^{-1}$, SIMBAD).

\section{Conclusion}

High quality rapid photometry data, obtained from one site reveal HD 23628 as a new $\delta$ Scuti object in the Pleiades cluster. The analysis reveals very low amplitude peaks, of the order of $1 \mathrm{mmag}$ and below. Four of them are detected with a $99 \%$ confidence level and are attributed to the new $\delta$ Scuti star. Some 

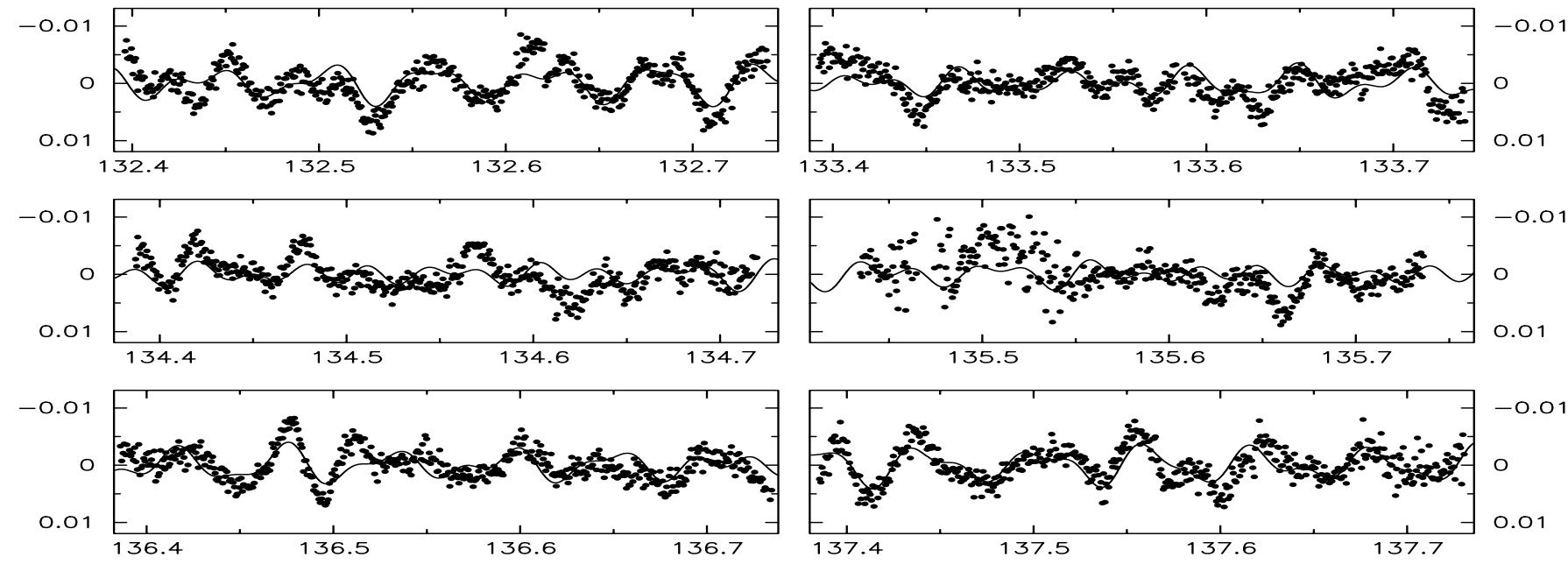

Fig. 3. The fit of the four-frequency solution to part of the data. Abscissa is the time in HJD $2451000+$ days, ordinate in magnitudes.

slightly lower amplitude pulsations are suspected. The fact that HD 23628 belongs to the Pleiades makes it a very interesting object for seismic interpretation, and we are considering new multisite observations in order to confirm and hopefully increase the list of its oscillation frequencies.

The discovery of this new low amplitude pulsator stresses the bias induced by classical observational detection limits in the understanding of the pulsation behaviour of $\delta$ Scuti objects. Space projects like COROT (Baglin et al. 2001), MOST (Matthews et al. 2001), MONS (Kjeldsen et al. 2000) or EDDINGTON (Gimenez \& Favata 2000) will bring a unique opportunity to break this detection limit by several orders of magnitude, shedding a new light on this field of research.

Acknowledgements. The STEPHI program has received financial support from the French CNRS, the Chinese National Natural Science Foundation and Academia Sinica, the Spanish DGES, the Mexican CONACYT. Special thanks are given to the staff of the observatories involved in the campaign. The $1.5 \mathrm{~m}$ Carlos Sánchez Telescope is operated on the island of Tenerife by the Instituto de Astrofísica de Canarias in the Spanish Observatorio del Teide.

\section{References}

Álvarez, M., Hernández, M. M., Michel, E., et al. 1998, A\&A, 340, 149

Baglin, A., Auvergne, M., Catala, C., et al. 2001, in Proceedings of the SOHO 10/GONG 2000 Workshop: Helio- and asteroseismology at the dawn of the millennium, ESA SP-464, 395

Breger, M. 1972, ApJ, 176, 367
Breger, M. 1973, A\&A, 22, 247

Breger, M. 1990, Delta Scuti Star Newsletter, 2, 13

Crawford, D. L., \& Perry, C. L. 1976, AJ, 81, 419

Crawford, D. L. 1979, AJ, 84, 1858

Cvetkovic, Z. 1992, A\&AS, 96, 191

Fox-Machado, L., Álvarez, M., Michel, E., et al. 2002, A\&A, 382, 556

Gatewood, et al. 2000, ApJ, 533, 938

Giménez, A., \& Favata, F. 2001, in Astrophysical ages and times scales, ASP Conf. Ser., 245, 304

Hernández, M. M., Michel, E., Belmonte, J. A., et al. 1998, A\&A, 337, 198

Kjeldsen, H., Bedding, T. R., \& Christensen-Dalsgaard, J. 2000, in The impact of large-scale surveys on pulsating stars research, ASP Conf. Ser., 203, 73

Li, Z.-P., Jiang, S.-Y., Liu, Y.-Y., \& Cao, M. 1991, A\&A, 245, 485

Matthews, J., Kuschnig, R., \& Shkolnik, E. 2001, in Proceedings of the SOHO 10/GONG 2000 Workshop: Helio- and asteroseismology at the dawn of the millennium, ESA SP-464, 385

Michel, E., Belmonte, J. A., Álvarez, M., et al. 1992, A\&A, 255, 139

Michel, E., Chevreton, M., Goupil, M. J., et al. 1995, in Proceedings of Fourth SOHO Workshop: Helioseismology, ESA SP-376, 533

Moon, T. T., \& Dworetsky, M. M. 1985, MNRAS, 217, 305

Nowroozi, A. A. 1967, Geophys. J. R. Astr. Soc., 12, 517

Rodríguez, E., López-González, M. J., López de Coca, P., et al. 2000, A\&AS, 144, 469

Roeser, S., \& Bastion, U. 1988, A\&AS, 74, 449

Sperl, M. 1998, Comm. in Astroseismology (Vienna), 111, 1

Suárez, J.-C., Michel, E., Houdek, G., et al. 2001, in Radial and nonradial pulsations as probes of stellar physics, ed. C. Aerts, T. R. Bedding, \& J. Christensen-Dalsgaard, ASP Conf. Ser., 259 Van Leeuwen, F. 1999, A\&A, 341, L71 